\title{
Internet Survey: Health Screening in Sports
}

\author{
Margaret Wang ${ }^{1}$, Gloria Wu², Vidhya Gunasekaran ${ }^{3}$, Victor $\mathrm{Chen}^{4}, \mathrm{Akash}_{\text {Vani }}{ }^{5}$, Don Kim ${ }^{6}$ \\ ${ }^{1}$ Harvard University. ${ }^{2}$ University of California, San Francisco. ${ }^{3}$ Aravind Eye Hospital. ${ }^{4}$ University of California, San \\ Diego, Division of Biological Sciences. ${ }^{5}$ University of California, Merced, Division of Biological Sciences. ${ }^{6}$ University of \\ California, Berkeley, Departments of Mathematics, Biological Sciences.
}

\begin{abstract}
While cardiovascular screening protocols exist, they have been focused on teenaged and college aged athletes versus adult athletes. To assess community awareness of health screening as related to adult athletes, we have created an internet questionnaire (QN). The survey was posted through social media (e.g. Facebook, Reddit, Flotrack and Active), for 11 months, that queried sports history, medical history, and symptoms while playing sports. A total of 3,750 respondents (R) answered the questionnaire, 2,776 male and 974 female. Age range: 18-83 yrs, avg: $33.7 \pm 11.22 \mathrm{yrs}$, median: $31 \mathrm{yrs}$. Seventy four per cent of $R(2,775 / 3,750)$ reported having at least one of the following symptoms while playing sports: dizziness, blacking/passing out, racing heartbeat, or chest pain, and $13.5 \%(505 / 3,750)$ of $R$ reported two or more. $62.3 \%(1,730 / 2,775)$ did not recall having symptoms. This underreporting was a result of "no one asking" $49.5 \%(857 / 1,730)$; "not answering after being asked" $28.2 \%(488 / 1,730)$, and "not telling the truth" $22.2 \%(384 / 1,730)$. Of interest, $97.1 \%(3,642 / 3,750)$ want a screening QN; 95.8\% $(3,592 / 3,750)$ want pre-screening by an MD. Prior to sports, only $22.9 \%(857 / 3,750)$ were required to answer a QN vs $38.0 \%(1,424 / 3,750)$ had a physical exam (PE); $14.9 \%(560 / 3,750)$ of individuals had both PE and QN. We conclude that adult participants in sports commonly experience symptoms but do not report them. Nearly all respondents favor participating in a screening questionnaire that would assess for cardiovascular problems.
\end{abstract}

\section{Introduction}

Screening protocols have been used to identify cardiovascular disorders in adult participants of sporting events, but these protocols are not uniformly administered in the various sports competitions such as marathons, triathlons, cycling races or endurance events. Atrial fibrillation (AF) is the most common cardiac arrhythmia. ${ }^{1}$

This internet survey aims to reach those adults who participate in any athletic activity. We would like to elicit responses about symptoms of cardiovascular disease among adult athletes.

While atrial fibrillation is the most common cardiac arrhythmia, the incidence of $\mathrm{AF}$ in adult athletes and its associated risk factors are not well studied. ${ }^{2}$ In the general population, AF is approximately $0.5 \%$ in men less than age 40 , and increases to $8 \%$ in men over age 80 years. Cardiac and non cardiac conditions such as age, structural

Key Words:

Sudden Cardiac Death, Social Media, Internet Survey, Athletes, High School Athletes, Recreational Athletes, Health Awareness, Health Screening.

Disclosures:

None.

Corresponding Author:

Gloria Wu,

Margaret Wang, 2550 Samaritan Drive,

Suite C, San Jose,

CA 95124. heart disease, diabetes, hypertension and hyperthyroidism have been implicated as risk factors in the development of AF. In patients younger than 60 years, routine evaluation has revealed no specific risk factors. ${ }^{2}$

One study showed that atrial fibrillation caused long-lasting palpitations in young elite athletes. ${ }^{3}$ Those with atrial fibrillation at a younger age had lower rates of mild hypertension compared to those who were not engaged in sports activity.

Studies have indicated the elevated risk of atrial fibrillation exists in adult athletes who participate in an intense exercise regimen such as marathons or more than 5-7 exercise sessions per week. ${ }^{3}$ Thus, recreational athletes may have an enhanced risk of developing cardiovascular problems via weekend sports competitions. This group of athletes have not been well studied.

Electrophysiological testing has been used as a method to identify risk of atrial fibrillation for athletes. ${ }^{4}$ The American Heart Association (AHA) recommends preparticipation screenings for athletes prior to athletic engagement. The AHA suggests that incorporating screening methods would identify risk for cardiovascular consequences, such as sudden cardiac death, nonfatal myocardial infarction, stroke, angina, acute coronary syndromes or heart failure. ${ }^{5}$

The purpose of our study was to assess the awareness of cardiac symptoms and symptom reporting in recreational and amateur sports participants via an internet survey. We chose the internet for its anonymity, its demographics and the possibility of reaching a large 
Age Distribution of Survey Respondents

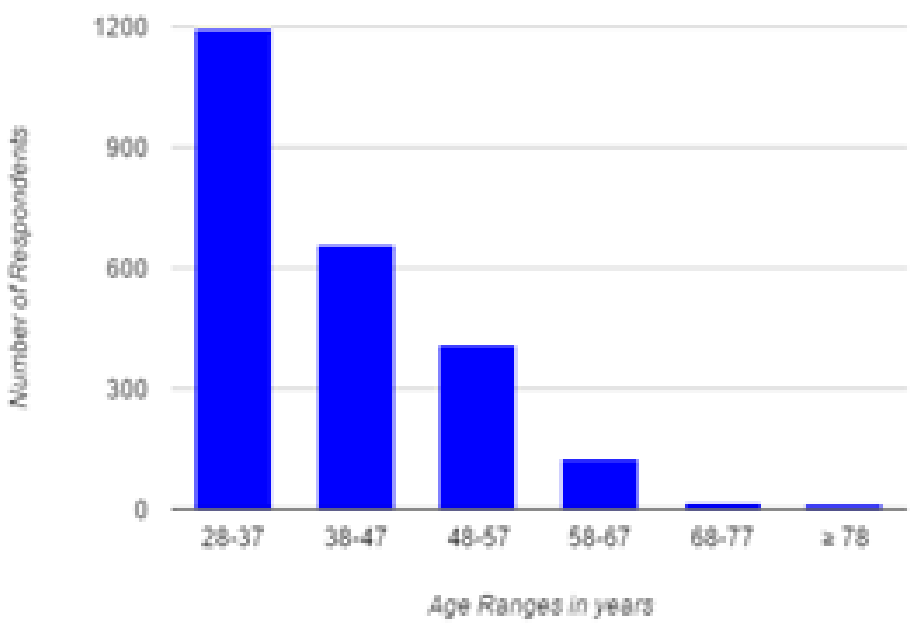

Figure 1: Age distribution of respondents to the survey

number of respondents. It may be important to identify the presence of cardiac conditions and arrhythmias in adult athletes through screening prior to athletic participation. Therefore, our aim was to evaluate the existence of cardiovascular symptoms in the general population through a social media survey.

\section{Material and Methods}

A 17-question survey was designed on Google Survey and distributed on various social media outlets and sports forums, such as Facebook, Reddit, Flotrak, and Active. Questions were designed to evaluate demographic factors, athletic history, and risk factors for

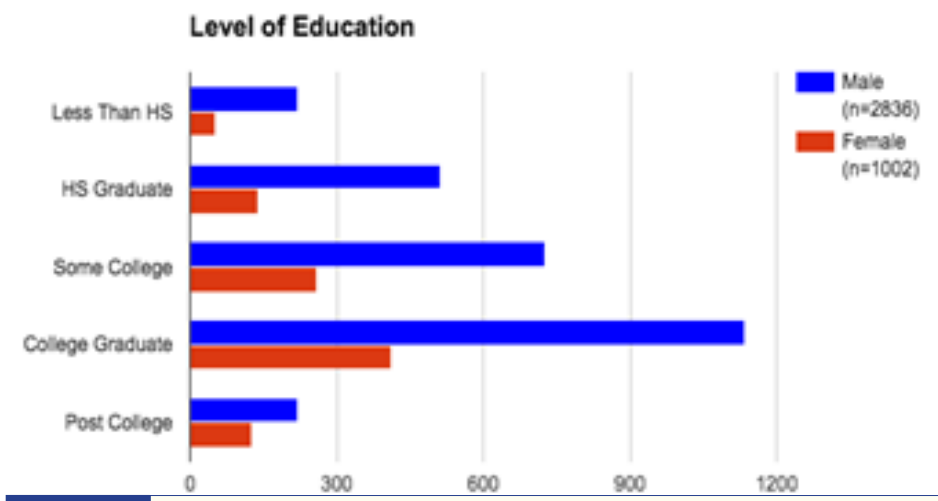

Figure 2: Highest level of education

cardiovascular disease (Table 1). The survey was reposted on these forums every day over the course of four months from October 24, 2014 to February 2015. Participants were required to be at least 18 years old, and informed consent was necessary in order to proceed with the survey.

Results

A total of 3,839 respondents answered the questionnaire, 2,837 male and 1002 female. $43.9 \%(1685 / 3839)$ of the population was under the age of 30 . Respondents ranged from ages 18 to 83 years old (mean: $33.7 \pm 11.22$ yrs), (Fig 1 ). The majority of respondents had college and post-college education (Fig 2). For country of origin/ ethnicity: United States, United Kingdom, India and Europe.

With respect to the level of athletic participation ( $\mathrm{Qn} 5$ ), the majority played in high school and college, (Fig 3). Most played sports for 2-5 years (Qn 6), (Fig 4). Most participated in years of 2001-2010 (Qn 7). Thus, most of the respondents participated in sports during the last decade (Fig 5).

Question 8 asks if the respondents had the following symptoms while participating in sports (Fig 6,7): $72.2 \%$ of the respondents $(2,775 / 3,839)$ reported having at least one of the following symptoms while playing sports: dizziness, blacking/passing out, racing heartbeat, or chest pain, and $13.2 \%(505 / 3,839)$ of respondents reported two or more symptoms.

Question 10 asks "Did you ever have symptoms while playing sports and not tell anyone? "Yes"responses totaled 1730; "No" responses totalled 1052. "Not applicable" totalled 726 responses.

Yet of symptomatic respondents who answered this question $(\mathrm{n}=1730)$ and of the 2775 who had one of symptoms in Qn $8(\mathrm{n}=2775)$ : $62.3 \%(1,730 / 2,775)$ did not report them. This underreporting noted in the answers of question 10 was a result of "no one asking" $49.5 \%$

\section{Qn 5: Level of Athletic Participation}

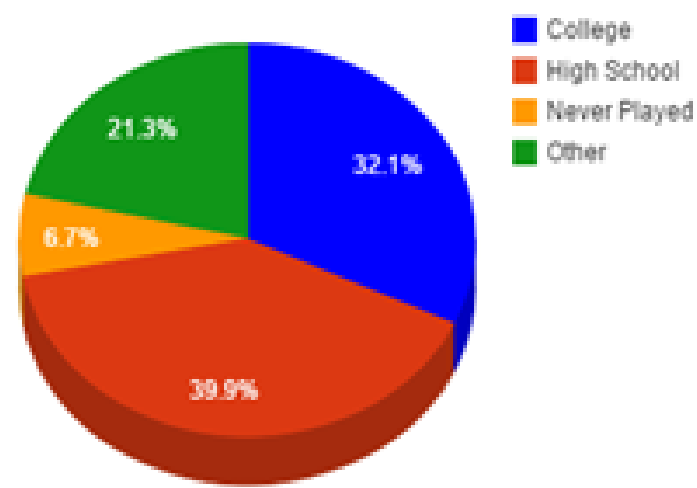

Figure 3: Level of most recent sports participation and competition

(857/1,730); "not answering after being asked" 28.2\% (488/1,730), and "not telling the truth" 22.2\% (384/1,730), (Fig 8).

For question 11: "Were you required to have a physical examination when you participated in sports," the majority of the respondents were not screened at all ( $\mathrm{n}=2139)$ (Fig 9). 246 respondents never played sports but answered this Internet Survey. Of note, 469 respondents were screened by their own doctor, 384 respondents were screened by the team doctor, 365 respondents were screened by the coach, 247 respondents were screened by the team athletic trainer (Fig 10).

Regarding question 13:"Have you ever been told that you have

\section{How many years did you participate?}

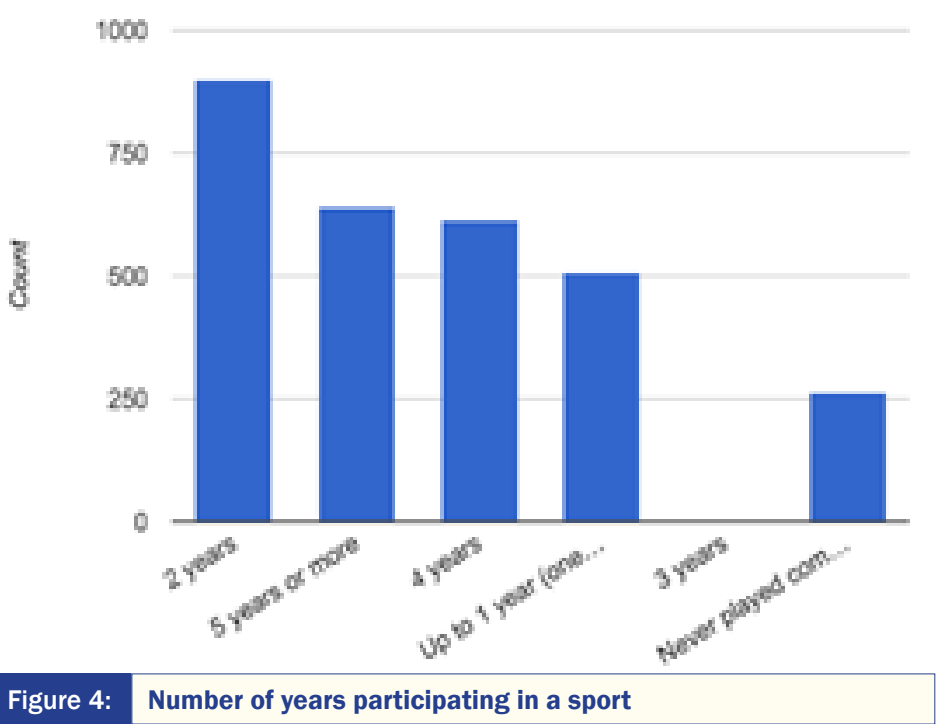

Figure 4: Number of years participating in a sport 


\section{When did you participate?}

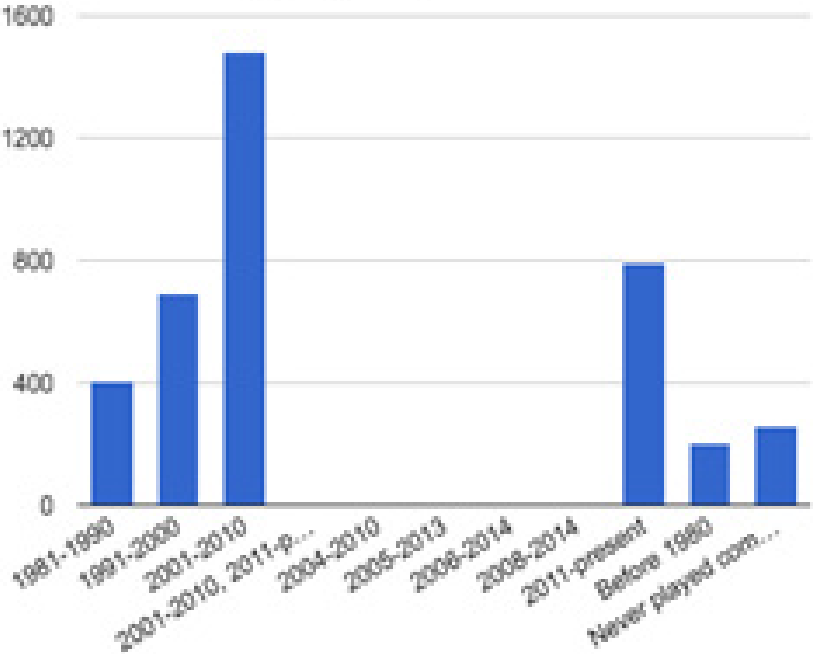

Figure 5: Sports participation by decade

an irregular heartbeat, a heart murmur, a heart condition, or a combination of the above?" The majority were never told of these conditions (Fig 11).

Question 14 deals with screening questionnaires: "Prior to sports participation, were you required to fill out a health screening questionnaire? $39.8 \%(1453 / 3839)$ were required to have a physical exam; $22.9 \%$ ( 880/3839)were required to complete a health screening questionnaire; $14.9 \%$ (573/3839) were required to have completed both screening questionnaire and physical exam.

\section{On 8: Reported Symptoms while Playing Sports}

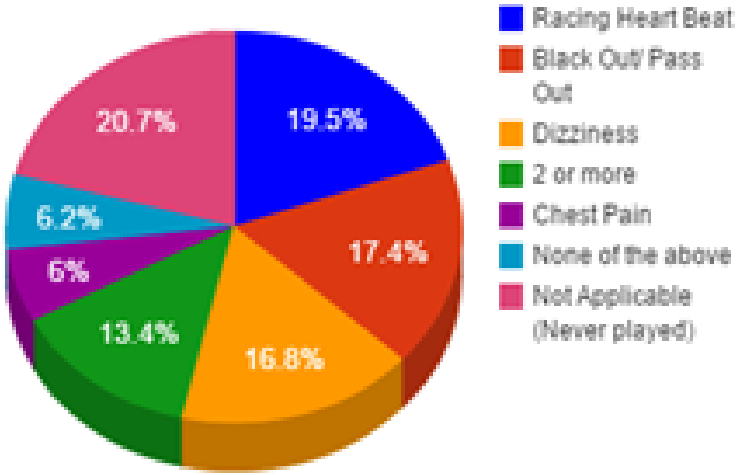

Figure 6:

Reported symptoms experienced by respondents while playing sports

For question 15: "Should health questionnaires be used before athletic competitions:" $95.0 \% \quad(3,642 / 3,839)$ prefer screening questionnaires prior to sports participation. For question 16: "Should physical exam by a medical be required before athletic competition?" $94 \% \%(3,592 / 3,839)$ would like pre-screening by an medical doctor. The responses to the last two questions suggests that there is strong public interest regarding preparticipation screening and examination for athletes (Fig 12).

\section{Discussion}

In our study population, the majority of individuals who experienced symptoms did not report them. While patient reliability is to be considered in these screening questionnaires, two-thirds of symptomatic respondents did not report their symptoms, and half did not report because "no one asked." These individuals with symptoms may be experiencing denial of the importance of these symptoms. The survey findings may demonstrate that individuals may not want to discontinue athletic participation despite having experienced symptoms. A known diagnosis may hinder an individual's enjoyment of sports as a hobby or recreational sports activities. A longitudinal study investigating arrhythmias in Olympic and World champion athletes found that temporary discontinuation of athletic activity could potentially eliminate atrial fibrillation. ${ }^{4}$

Intense exercise is linked to cardiovascular changes that may contribute to the development of AF. ${ }^{3,6,7}$ Intense sports involvement contributes to vagal tone and increased risk of atrial fibrillation. Competitive athletes show left atrial enlargement due to left ventricular cavity enlargement. ${ }^{6}$ Another study demonstrated that

\section{Qn 9: Did you ever have symptoms and not tell anyone?}

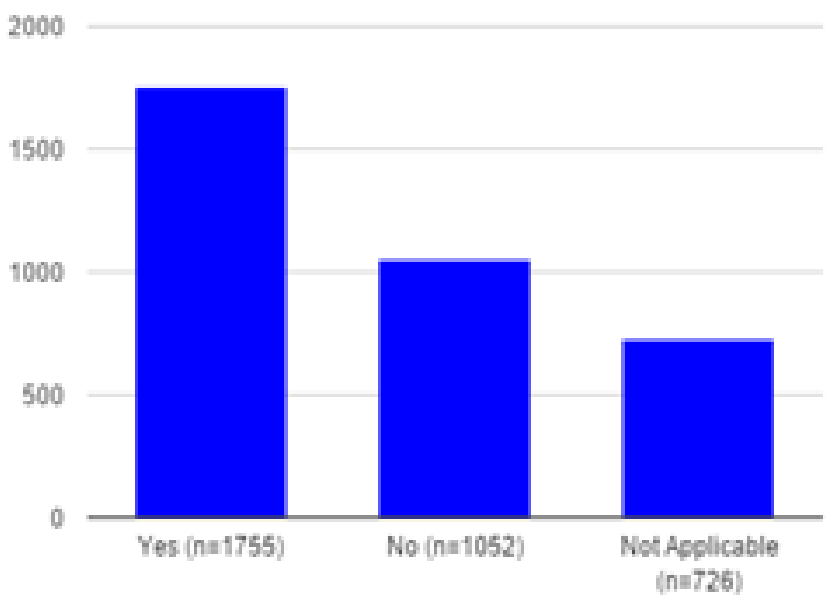

Figure 7: Reporting the presence of symptoms

those who participated in frequent exercise had greater ventricular mass, as well as larger atrial and ventricular dimensions. ${ }^{7}$ These studies support that intense exercise enhances the risk of $\mathrm{AF}^{3,6,7}$

For individuals over age 50 and participate in rigorous activity, the risk of developing AF decreases. People who exercised five to seven times per week increased their AF risk by $20 \%$, by $53 \%$ if they jogged, compared to individuals who were sedentary. Recreational athletes

\section{Qn 10: Rates of Symptom Reporting}

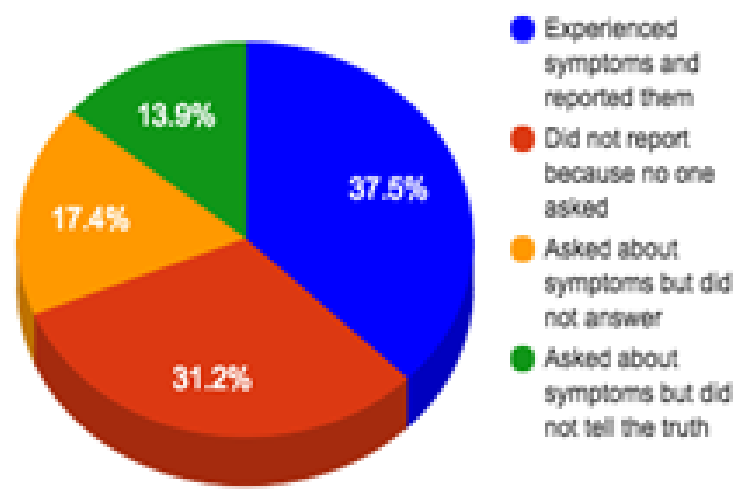

Combined data from Qn 9, 10 suggests that public response was not truth-based and/or their athletic organizers/staff never asked the appropriate questions when the respondents were asked about their symptoms 


\section{Were you required to have a physical examination before you participated in the sport?}

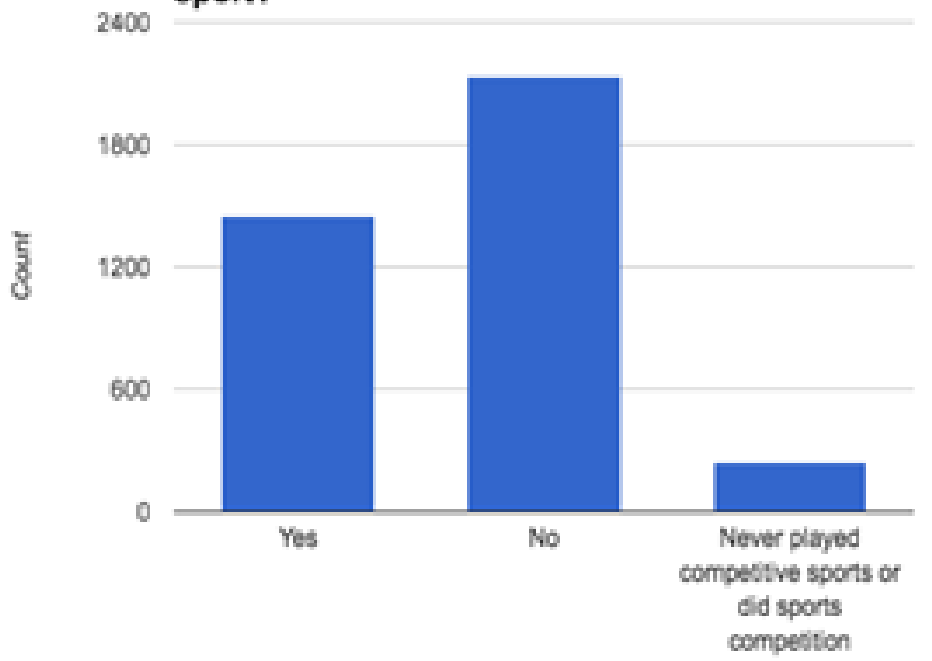

Figure 9: Physical examination requirement prior to sports participation

have differing levels of training and stressors on their cardiovascular systems. The type of exercise has an impact on risk of developing $\mathrm{AF}^{3}$ Engagement in dynamic exercise leads to enhanced dimensions of heart cavities, whereas static exercise results in hypertrophy. ${ }^{7}$ This suggests that adult athletes are at risk for developing $\mathrm{AF}$ and all adults who participate in regular sports competitions might benefit from cardiovascular screenings according to the AHA guidelines.

Limitations in our study are that this is an internet based study and the responses are skewed towards younger adults, male preponderance. Thus, it may be difficult to generalize about the entire adult athletic community which would include college athletes, professional athletes, occasional athletes, adult sports league participants, and adult competitors who are non-professional athletes and older/senior athletes Not all of these adult athletes would be found in the internet blog and forum sites where we posted the questionnaire. Due to the demographics of the forums where the survey was distributed, the study population may have been particularly interested in sports and health. This group's enthusiasm for preparticipation screening and physical exams may be a reflection of the self-selected group of people who like to participate in sports and athletic blogs. Respondents also needed a computer and access to internet to complete the survey, suggesting a higher education and socioeconomic status.

\section{Qn 12: Physical Exam}

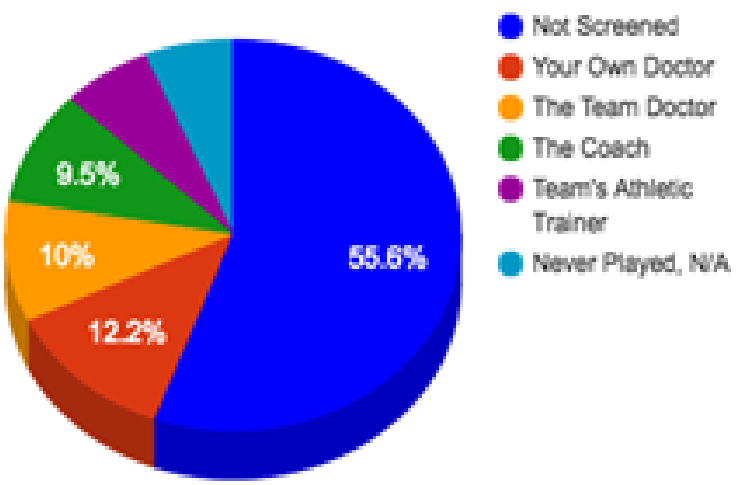

Figure 10:

Whether or not the individual was screened by physical exam. If screened, who did the screening

\section{Have you ever been told that you have...}

2000

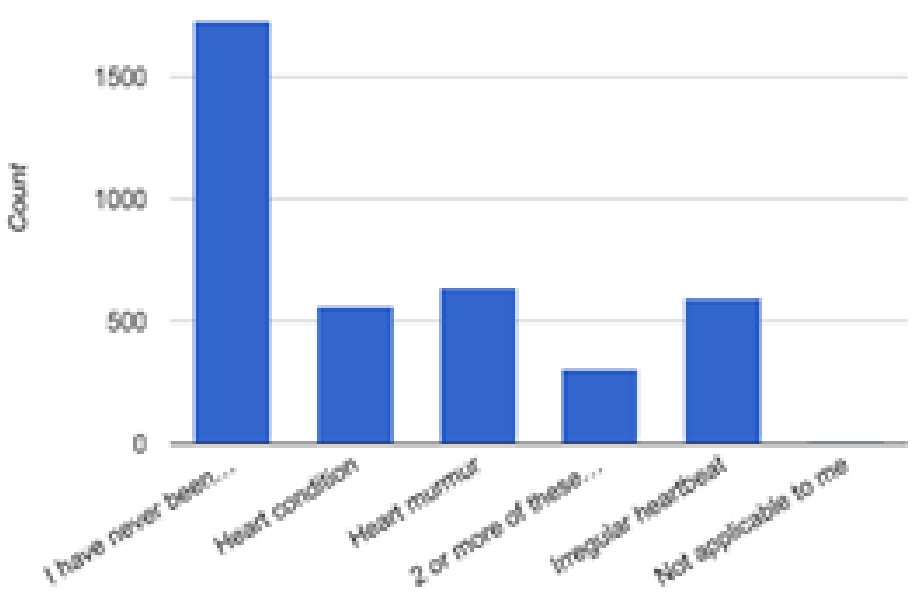

Figure 11: Reporting of symptoms

While this survey was skewed towards the young and educated demographic with access to the internet, these findings could guide us towards further discussion about health screening in sports among cardiologists and primary care physicians. In our study we identified a high proportion of reported symptoms, including racing heartbeat, unconsciousness, dizziness, and chest pain. A majority of those who exhibited cardiac-like symptoms, more than half, did not report them to their MDs, RNs, coaches or trainers because "no one asked." The results from our study suggest that patients could benefit from being informed on the impact of frequent exercise on cardiovascular disease.

\section{Conclusions}

In our study, the respondents reported the presence of cardiac symptoms related to and separate from athletic participation. Despite the screening standards provided by the American Heart Association, pre-screening is not performed consistently for high school and recreational athletes. The responses from our Internet survey show that more public education is needed since there are no formal screening protocols that are uniformly applied to high school and recreational athletes. There is almost unanimous desire among the respondents for screening and physical examinations prior to athletic events. As a first step, advocating for greater awareness of the American Heart Association guidelines among all physicians would help in the effort of public education for Sudden Cardiac Death.

\section{References}

1. Mozaffarian Dariush, FurbergCurt D, PsatyBruce M, SiscovickDavid. Physical activity and incidence of atrial fibrillation in older adults: the cardiovascular health study. Circulation. 2008;118 (8):800-7.

2. Calvo Naiara, BrugadaJosep, SitgesMarta, MontLluís. Atrial fibrillation and atrial flutter in athletes. Br J Sports Med. 2012;46 Suppl 1:i37-43.

3. Aizer Anthony, GazianoJ Michael, CookNancy R, MansonJoann E, BuringJulie $\mathrm{E}$, AlbertChristine M. Relation of vigorous exercise to risk of atrial fibrillation. Am. J. Cardiol. 2009;103 (11):1572-7.

4. Furlanello F, BertoldiA, DallagoM, GalassiA, FernandoF, BiffiA, MazzoneP, PapponeC, ChierchiaS. Atrial fibrillation in elite athletes. J. Cardiovasc. Electrophysiol. 1998;9 (8 Suppl):S63-8.

5. Maron B J, AraújoC G, ThompsonP D, FletcherG F, de LunaA B, FlegJ L, PellicciaA, BaladyG J, FurlanelloF, Van CampS P, ElosuaR, ChaitmanB $\mathrm{R}$, BazzarreT L. Recommendations for preparticipation screening and the 
assessment of cardiovascular disease in masters athletes: an advisory for healthcare professionals from the working groups of the World Heart Federation, the International Federation of Sports Medicine, and the American Heart Association Committee on Exercise, Cardiac Rehabilitation, and Prevention. Circulation. 2001;103 (2):327-34.

6. Pelliccia Antonio, MaronBarry J, Di PaoloFernando M, BiffiAlessandro, QuattriniFilippo M, PisicchioCataldo, RoselliAlessandra, CaselliStefano, CulassoFranco. Prevalence and clinical significance of left atrial remodeling in competitive athletes. J. Am. Coll. Cardiol. 2005;46 (4):690-6.

7. Mont L, SambolaA, BrugadaJ, VaccaM, MarrugatJ, ElosuaR, ParéC, AzquetaM, SanzG. Long-lasting sport practice and lone atrial fibrillation. Eur. Heart J. 2002;23 (6):477-82. 